\title{
Fully heavy pentaquarks in quark models
}

\author{
Ye Yan, ${ }^{\S}$ Yuheng Wu, ${ }^{\|}$Xiaohuang Hu, ${ }^{\star}$ Hongxia Huang $\odot,{ }^{*}$ and Jialun Ping ${ }^{\dagger}$ \\ Department of Physics, Nanjing Normal University, Nanjing, Jiangsu 210097, People's Republic of China
}

(Received 22 October 2021; accepted 10 January 2022; published 26 January 2022)

\begin{abstract}
The fully heavy pentaquarks $c c c c \bar{c}$ and $b b b b \bar{b}$ are systematically investigated within the chiral quark model and quark delocalization color screening model. The results are consistent in both of the models, and the effect of the channel coupling is crucial for forming a bound state of the fully heavy pentaquark system. Three possible fully heavy pentaquarks are obtained and are the $c c c c \bar{c}$ state with $J^{P}=1 / 2^{-}$and the mass of 7891.9-7892.7 MeV, the $b b b b \bar{b}$ state with $J^{P}=1 / 2^{-}$and the mass of $23810.1-23813.8 \mathrm{MeV}$, and the $b b b b \bar{b}$ state with $J^{P}=3 / 2^{-}$and the mass of $23748.2-23752.3 \mathrm{MeV}$. All these fully heavy pentaquark states are worth searching in future experiments.
\end{abstract}

DOI: 10.1103/PhysRevD.105.014027

\section{INTRODUCTION}

Over the past decades, great progress has been made on studying exotic states, among which the fully heavy tetraquark states $(Q Q \bar{Q} \bar{Q}, Q=c, b)$ attracted extensive attention, since such states with very large energy can be accessed experimentally and easily distinguished from other states. In 2020, the LHCb Collaboration reported their result on the observation of the fully charm state $(c c \bar{c} \bar{c})$. A narrow structure $X(6900)$, matching the line shape of a resonance and a broad structure next to the $J / \psi J / \psi$ mass threshold, was obtained [1]. Theoretically, this fully charm tetraquark has been investigated in the framework of the QCD sum rules [2], the potential model [3], the nonrelativistic diquark-antidiquark model [4], the relativized quark model [5], string junction picture [6], and the constituent quark model $[7,8]$. Meanwhile, the molecular configurations for the fully charm tetraquark states were proposed by the perturbation QCD [9], while the compact structure was obtained in the holography inspired stringy hadron model [10]. In the recently theoretical investigations, the experimental data and theoretical importance on the $c c \bar{c} \bar{c}$ tetraquarks were reviewed in the articles [11,12].

\footnotetext{
*Corresponding author. hxhuang@njnu.edu.cn

Corresponding author. jlping@njnu.edu.cn

\$201001002@njnu.edu.cn

\$201002013@njnu.edu.cn

"191002007@njnu.edu.cn
}

Published by the American Physical Society under the terms of the Creative Commons Attribution 4.0 International license. Further distribution of this work must maintain attribution to the author(s) and the published article's title, journal citation, and DOI. Funded by SCOAP ${ }^{3}$.
For the dibaryons, although the deuteron is the only stable state composed of two nucleons, there are possible bound or resonant dibaryons with and without strange quarks. Both the high strangeness dibaryons $N \Omega$ and $\Omega \Omega$ were proposed by quark models $[13,14]$. The lattice QCD simulations by HAL QCD Collaboration have studied the six-quark systems containing light or strange quarks and confirmed the existence of the $N \Omega$ and $\Omega \Omega$ bound states with nearly physical quark masses $\left(m_{\pi} \simeq 146 \mathrm{MeV}\right.$ and $m_{K} \simeq 525 \mathrm{MeV}$ ) $[15,16]$. The fully heavy dibaryons were also proposed by both the quark models [17] and the lattice QCD method [18,19], in which the dibaryon with the highest charm number $\Omega_{c c c} \Omega_{c c c}$ was possible to exist.

Regarding the pentaquarks, the most noteworthy states in recent years are the hidden-charm pentaquarks. In 2015, the $\mathrm{LHCb}$ Collaboration reported two hidden-charm pentaquark states $P_{c}(4380)$ and $P_{c}(4450)$ in the $J / \psi p$ invariant mass spectrum of $\Lambda_{b}^{0} \rightarrow J / \psi K^{-} p$ [20]. Four years later, the $\mathrm{LHCb}$ Collaboration updated their results, a new state $P_{c}(4312)$ was proposed, and the $P_{c}(4450)$ was split into $P_{c}(4440)$ and $P_{c}(4457)$ states [21]. These observations of the hidden-charm pentaquarks bring great interest in theoretical investigations. Excellent review on the hidden-charm pentaquarks can be found in Refs. [22-24].

Accordingly, it is timely to study the existence of the fully heavy pentaquarks. A systematic study on the mass spectra of the $S$-wave fully heavy pentaquarks $Q Q Q Q \bar{Q}$ has been performed in the framework of the chromomagnetic interaction (CMI) model [25]. Besides, the fully heavy pentaquarks were also investigated by the QCD sum rule approach [26,27].

The QCD is the underlying theory of the strong interaction. However, it is difficult to study the structure of the hadrons and the hadron-hadron interaction directly because of the nonperturbative properties of QCD in the 
low-energy region. Although lattice QCD has made impressive progress on nucleon-nucleon interactions and multiquark systems [28-30], the QCD-inspired quark model is still the main approach to study the hadronhadron interactions and multiquark states. A common approach is the chiral quark model (ChQM) [31], in which the constituent quarks interact with each other through colorless Goldstone bosons exchange in addition to the colorful one-gluon exchange and confinement, and the chiral partner $\sigma$ meson-exchange is introduced to obtain the immediate-range attraction of hadron-hadron interaction. An alternative approach is the quark delocalization color screening model (QDCSM), which was developed in the 1990s with the aim of explaining the similarities between nuclear and molecular forces [32]. Both of the models have been successfully applied to the study of the properties of deuteron, nucleon-nucleon, and hyperonnucleon interactions [33,34]. Recently, these two models were used to study the fully heavy tetraquarks [8] and dibaryons [17]. Therefore, it is interesting to extend these two models to the fully heavy pentaquarks.

This paper is organized as follows. First, both the chiral quark model and the quark delocalization color screening model are introduced briefly in Sec. II. The way we construct the wave functions is presented, too. Then, the numerical results and discussions are given in Sec. III. Finally, the summary is given in Sec. IV.

\section{MODEL AND WAVE FUNCTIONS}

In this work, we use two models, ChQM and QDCSM, to investigate the fully heavy pentaquark systems. In this sector, we will introduce these two models and the wave functions of the pentaquark systems.

\section{A. Chiral quark model}

The Salamanca version was chosen as the representative of the chiral quark models because the Salamanca group's work covers the hadron spectra, nucleon-nucleon interaction, and multiquark states. The model details can be found in Ref. [31]. Here, we only give the Hamiltonian,

$H=\sum_{i=1}^{5}\left(m_{i}+\frac{p_{i}^{2}}{2 m_{i}}\right)-T_{c m}+\sum_{j>i=1}^{5}\left(V_{i j}^{\mathrm{CON}}+V_{i j}^{\mathrm{OGE}}\right)$, where $T_{c m}$ is the kinetic energy of the center of mass; $V_{i j}^{\mathrm{CON}}$ and $V_{i j}^{\text {OGE }}$ are the interactions of the confinement and the one-gluon-exchange, respectively. For the fully heavy systems, there is no $\sigma$ exchange nor the Goldstone boson exchange. The central parts of $V_{i j}^{\mathrm{CON}}$ and $V_{i j}^{\mathrm{OGE}}$ are

$$
V_{i j}^{\mathrm{CON}}=-\lambda_{i}^{c} \cdot \lambda_{j}^{c}\left(a_{c} r_{i j}^{2}+V_{0}\right)
$$

$V_{i j}^{\mathrm{OGE}}=\frac{\alpha_{s}}{4} \lambda_{i}^{c} \cdot \lambda_{j}^{c}\left[\frac{1}{r_{i j}}-\frac{\pi}{2} \delta\left(\mathbf{r}_{i j}\right)\left(\frac{1}{m_{i}^{2}}+\frac{1}{m_{j}^{2}}+\frac{4 \sigma_{i} \cdot \sigma_{j}}{3 m_{i} m_{j}}\right)\right]$,

where $\alpha_{s}$ is the quark-gluon coupling constant. To cover the wide energy range from light to strange to heavy quarks, an effective scale-dependent quark-gluon coupling $\alpha_{s}(u)$ was introduced [35]:

$$
\alpha_{s}(u)=\frac{\alpha_{0}}{\ln \left(\frac{u^{2}+u_{0}^{2}}{\Lambda_{0}^{2}}\right)} .
$$

The other symbols in the above expressions have their usual meanings. All parameters, which are fixed by fitting to the masses of baryons with light flavors and heavy flavors, are taken from our previous work Ref. [36].

\section{B. Quark delocalization color screening model}

Generally, the Hamiltonian of QDCSM is almost the same as that of ChQM, but with two modifications [32]. One is that the $\sigma$-exchange no longer exists in QDCSM, the other is that the screened color confinement is used in QDCSM between quark pairs reside in different clusters, aimed to take into account the QCD effect which has not yet been included in the two-body confinement and effective one-gluon exchange. Since there is no $\sigma$-meson exchange interaction between the heavy quarks (c or b), the only difference here is the confinement interaction. The confining potential in QDCSM was modified as

$$
V_{i j}^{\mathrm{CON}}= \begin{cases}-\lambda_{i}^{c} \cdot \lambda_{j}^{c}\left(a_{c} r_{i j}^{2}+V_{0}\right), & \mathrm{i}, \mathrm{j} \text { in the same cluster } \\ -\lambda_{i}^{c} \cdot \lambda_{j}^{c}\left(a_{c} \frac{1-e^{-\mu q_{i} q_{j} r_{i j}^{2}}}{\mu_{q_{i} q_{j}}}+V_{0}\right), & \text { otherwise, }\end{cases}
$$

where $\mu_{q_{i} q_{j}}$ is the color screening parameter, which is related to the flavor of the $i$ th quark $q_{i}$ and the $j$ th quark $q_{j}$. For the light quark systems, $\mu_{u u}, \mu_{u s}$, and $\mu_{s s}$ are determined by fitting the deuteron properties, nucleon-nucleon scattering phase shifts, and hyperon-nucleon scattering phase shifts, respectively, with $\mu_{u u}=0.45 \mathrm{fm}^{-2}, \mu_{u s}=0.19 \mathrm{fm}^{-2}$, and $\mu_{s s}=0.08 \mathrm{fm}^{-2}$, satisfying the relation, $\mu_{u s}^{2}=\mu_{u u} \mu_{s s}$ [34]. Besides, we found that the heavier the quark, the smaller this 
parameter $\mu_{q_{i} q_{j}}$. When extending to the heavy quark system, the hidden-charm pentaqyark system, we took $\mu_{c c}$ as a adjustable parameter from 0.01 to $0.001 \mathrm{fm}^{-2}$ and found that the results were insensitive to the value of $\mu_{c c}$ [37] and the $P_{c}$ states were well predicted in the work of Refs [37,38]. In this work, we investigate the fully heavy pentaquarks $\operatorname{cccc} \bar{c}$ and $b b b b \bar{b}$, and we also take $\mu_{c c}$ and $\mu_{b b}$ as adjustable parameters, which are $\mu_{c c}=0.01 \sim 0.001 \mathrm{fm}^{-2}$ and $\mu_{b b}=0.001 \sim 0.0001 \mathrm{fm}^{-2}$, respectively. We find the results are also insensitive to the value of $\mu_{c c}$ and $\mu_{b b}$. In fact, if the value of the $\mu_{q_{i} q_{j}}$ is very small, the exponential function can be approximated to

$$
e^{-\mu_{q_{i} q_{j}} r_{i j}^{2}}=1-\mu_{q_{i} q_{j}} r_{i j}^{2}+\cdots
$$

According to Eq. (6), the second expression in Eq. (5) can be rewritten as

$$
\begin{aligned}
V_{i j}^{\mathrm{CON}} & =-\lambda_{i}^{c} \cdot \lambda_{j}^{c}\left(a_{c} \frac{1-e^{-\mu_{q_{i} q_{j}} r_{i j}^{2}}}{\mu_{q_{i} q_{j}}}+V_{0}\right) \\
& \approx-\lambda_{i}^{c} \cdot \lambda_{j}^{c}\left(a_{c} r_{i j}^{2}+V_{0}\right)
\end{aligned}
$$

which is the same with the expression of two quarks in the same cluster. So, when the value of the $\mu_{q_{i} q_{j}}$ is very small, the screened confinement will return to the quadratic form, which is why the results are insensitive to the value of $\mu_{c c}$ and $\mu_{b b}$. So, in the present work, we show the results by using $\mu_{c c}=0.01 \mathrm{fm}^{-2}$ and $\mu_{b b}=0.001 \mathrm{fm}^{-2}$.

\section{Wave functions}

The resonating group method (RGM) $[39,40]$ and generating coordinates method $[41,42]$ are used to carry out a dynamical calculation. The main feature of the RGM for two-cluster systems is that it assumes that two clusters are frozen inside and only considers the relative motion between the two clusters. So, the conventional ansatz for the two-cluster wave functions is

$$
\psi_{5 q}=\mathcal{A}\left[\left[\phi_{B_{1}} \phi_{B_{2}}\right]^{[\sigma] I S} \otimes \chi_{L}(\boldsymbol{R})\right]^{J},
$$

where the symbol $\mathcal{A}$ is the antisymmetrization operator and $\mathcal{A}=1-P_{14}-P_{24}-P_{34} \cdot[\sigma]=[222]$ gives the total color symmetry and all other symbols have their usual meanings. $\phi_{B_{1}}$ and $\phi_{B_{2}}$ are the $q^{3}$ and $q \bar{q}$ cluster wave functions, respectively. From the variational principle, after variation with respect to the relative motion wave function $\chi(\mathbf{R})=\sum_{L} \chi_{L}(\mathbf{R})$, one obtains the RGM equation

$$
\int H\left(\mathbf{R}, \mathbf{R}^{\prime}\right) \chi\left(\mathbf{R}^{\prime}\right) d \mathbf{R}^{\prime}=E \int N\left(\mathbf{R}, \mathbf{R}^{\prime}\right) \chi\left(\mathbf{R}^{\prime}\right) d \mathbf{R}^{\prime},
$$

where $H\left(\mathbf{R}, \mathbf{R}^{\prime}\right)$ and $N\left(\mathbf{R}, \mathbf{R}^{\prime}\right)$ are Hamiltonian and norm kernels. By solving the RGM equation, we can get the energies $E$ and the wave functions. In fact, it is not convenient to work with the RGM expressions. Then, we expand the relative motion wave function $\chi(\mathbf{R})$ by using a set of Gaussians with different centers,

$$
\begin{aligned}
\chi_{L}(\boldsymbol{R})= & \frac{1}{\sqrt{4 \pi}}\left(\frac{6}{5 \pi b^{2}}\right)^{3 / 4} \sum_{i=1}^{n} C_{i} \\
& \times \int \exp \left[-\frac{3}{5 b^{2}}\left(\boldsymbol{R}-\boldsymbol{S}_{i}\right)^{2}\right] Y^{L}\left(\hat{\boldsymbol{S}}_{i}\right) d \hat{\boldsymbol{S}}_{i},
\end{aligned}
$$

where $L$ is the orbital angular momentum between two clusters and $S_{i}, i=1,2, \ldots, n$ are the generator coordinates, which are introduced to expand the relative motion wave function. By including the center of mass motion,

$$
\phi_{C}\left(\boldsymbol{R}_{C}\right)=\left(\frac{5}{\pi b^{2}}\right)^{3 / 4} e^{-\frac{5 R_{C}^{2}}{2 b^{2}}},
$$

the ansatz (8) can be rewritten as

$$
\begin{aligned}
\psi_{5 q}= & \mathcal{A} \sum_{i=1}^{n} C_{i} \int \frac{d \hat{\boldsymbol{S}}_{i}}{\sqrt{4 \pi}} \prod_{\alpha=1}^{3} \phi_{\alpha}\left(\boldsymbol{S}_{i}\right) \prod_{\beta=4}^{5} \phi_{\beta}\left(-\boldsymbol{S}_{i}\right) \\
& \times\left[\left[\chi_{I_{1} S_{1}}\left(B_{1}\right) \chi_{I_{2} S_{2}}\left(B_{2}\right)\right]^{I S} Y_{L M}\left(\hat{\boldsymbol{S}}_{i}\right)\right]^{J} \\
& \times\left[\chi_{c}\left(B_{1}\right) \chi_{c}\left(B_{2}\right)\right]^{[\sigma]},
\end{aligned}
$$

where $\chi_{I_{1} S_{1}}$ and $\chi_{I_{2} S_{2}}$ are the products of the flavor and spin wave functions and $\chi_{c}$ is the color wave function. These will be shown in detail later. $\phi_{\alpha}\left(\boldsymbol{S}_{i}\right)$ and $\phi_{\beta}\left(-\boldsymbol{S}_{i}\right)$ are the single-particle orbital wave functions with different reference centers:

$$
\begin{aligned}
\phi_{\alpha}\left(\boldsymbol{S}_{i}\right) & =\left(\frac{1}{\pi b^{2}}\right)^{3 / 4} e^{-\frac{1}{2 b^{2}}\left(r_{\alpha}-\frac{2}{5} S_{i}\right)^{2}} \\
\phi_{\beta}\left(-S_{i}\right) & =\left(\frac{1}{\pi b^{2}}\right)^{3 / 4} e^{-\frac{1}{2 b^{2}}\left(r_{\beta}+\frac{3}{5} S_{i}\right)^{2}} .
\end{aligned}
$$

With the reformulated ansatz (12), the RGM equation (9) becomes an algebraic eigenvalue equation,

$$
\sum_{j} C_{j} H_{i, j}=E \sum_{j} C_{j} N_{i, j}
$$

where $H_{i, j}$ and $N_{i, j}$ are the Hamiltonian matrix elements and overlaps, respectively. By solving the generalized eigenproblem, we can obtain the energy and the corresponding wave functions of the pentaquark system.

The quark delocalization in QDCSM is realized by specifying the single-particle orbital wave function of QDCSM as a linear combination of left and right Gaussians, which are the single-particle orbital wave functions used in the ordinary quark cluster model, 


$$
\begin{aligned}
\psi_{\alpha}\left(\boldsymbol{S}_{i}, \epsilon\right) & =\left(\phi_{\alpha}\left(\boldsymbol{S}_{\boldsymbol{i}}\right)+\epsilon \phi_{\alpha}\left(-\boldsymbol{S}_{\boldsymbol{i}}\right)\right) / N(\epsilon), \\
\psi_{\beta}\left(-\boldsymbol{S}_{i}, \epsilon\right) & =\left(\phi_{\beta}\left(-\boldsymbol{S}_{\boldsymbol{i}}\right)+\epsilon \phi_{\beta}\left(\boldsymbol{S}_{\boldsymbol{i}}\right)\right) / N(\epsilon), \\
N\left(S_{i}, \epsilon\right) & =\sqrt{1+\epsilon^{2}+2 \epsilon e^{-S_{i}^{2} / 4 b^{2}}} .
\end{aligned}
$$

Here, the mixing parameter $\epsilon$ is not an adjusted one but determined variationally by the dynamics of the multiquark system itself. In this way, the multiquark system chooses its favorable configuration in the interacting process. This mechanism has been used to explain the crossover transition between hadron phase and quark-gluon plasma phase [43].

For the spin wave function, we first construct the spin wave function of the $q^{3}$ and $q \bar{q}$ clusters, and then the total spin wave function of the pentaquark system is obtained by coupling the spin wave functions of two clusters together. The spin wave functions of the $q^{3}$ and $q \bar{q}$ clusters are

$$
\begin{aligned}
\chi_{\frac{3}{2}, \frac{3}{2}}^{\sigma}(3) & =\alpha \alpha \alpha \\
\chi_{\frac{3}{2}, \frac{1}{2}}^{\sigma}(3) & =\frac{1}{\sqrt{3}}(\alpha \alpha \beta+\alpha \beta \alpha+\beta \alpha \alpha) \\
\chi_{\frac{3}{2},-\frac{1}{2}}^{\sigma}(3) & =\frac{1}{\sqrt{3}}(\alpha \beta \beta+\beta \alpha \beta+\beta \beta \alpha) \\
\chi_{1,1}^{\sigma}(2) & =\alpha \alpha \\
\chi_{1,0}^{\sigma}(2) & =\frac{1}{\sqrt{2}}(\alpha \beta+\beta \alpha) \\
\chi_{1,-1}^{\sigma}(2) & =\beta \beta \\
\chi_{0,0}^{\sigma}(2) & =\frac{1}{\sqrt{2}}(\alpha \beta-\beta \alpha) .
\end{aligned}
$$

For the pentaquark system, the total spin quantum number can be $1 / 2,3 / 2$, or $5 / 2$, so the wave function of each spin quantum number can be written as follows:

$$
\begin{aligned}
\chi_{\frac{1}{2}, \frac{1}{2}}^{\sigma 1}(5)= & \sqrt{\frac{1}{6}} \chi_{\frac{3}{2},-\frac{1}{2}}^{\sigma}(3) \chi_{1,1}^{\sigma}(2)-\sqrt{\frac{1}{3}} \chi_{\frac{3}{2}, \frac{1}{2}}^{\sigma}(3) \chi_{1,0}^{\sigma}(2) \\
& +\sqrt{\frac{1}{2}} \chi_{\frac{3}{2}, \frac{3}{2}}^{\sigma}(3) \chi_{1,-1}^{\sigma}(2) \\
\chi_{\frac{3}{2}, \frac{3}{2}}^{\sigma 1}(5)= & \sqrt{\frac{3}{5}} \chi_{\frac{3}{2}, \frac{3}{2}}^{\sigma}(3) \chi_{1,0}^{\sigma}(2)-\sqrt{\frac{2}{5}} \chi_{\frac{3}{2}, \frac{1}{2}}^{\sigma}(3) \chi_{1,1}^{\sigma}(2) \\
\chi_{\frac{3}{2}, \frac{3}{2}}^{\sigma 2}(5)= & \chi_{\frac{3}{2}, \frac{3}{2}}^{\sigma}(3) \chi_{0,0}^{\sigma}(2) \\
\chi_{\frac{5}{2}, \frac{5}{2}}^{\sigma}(5)= & \chi_{\frac{3}{2}, \frac{3}{2}}^{\sigma}(3) \chi_{1,1}^{\sigma}(2) .
\end{aligned}
$$

The flavor wave functions for the fully heavy pentaquark system we investigate here are very simple and are

$$
\begin{gathered}
\chi_{00}^{f m 1}=\operatorname{cccc} \bar{c} \\
\chi_{00}^{f m 2}=b b b b \bar{b} .
\end{gathered}
$$

The construction of the color wave function is similar to the spin wave function. We first write down the color wave function of the $q^{3}$ and $q \bar{q}$ clusters, and then the color wave function of the pentaquark system is obtained by coupling the two clusters together. For the color-singlet channel (two clusters are color singlet), the color wave function is

$$
\begin{aligned}
\chi^{c 0}= & \frac{1}{\sqrt{6}}(r g b-r b g+g b r-g r b+b r g-b g r) \\
& \times \frac{1}{\sqrt{3}}(r \bar{r}+g \bar{g}+b \bar{b}) .
\end{aligned}
$$

For the hidden-color channel (two clusters are color octet), the symmetry of the color-octet cluster is [21]. The color wave function of the color-octet $q^{3}$ cluster can be written as

$$
\begin{aligned}
& \chi_{b 1}^{c 1}=\frac{1}{\sqrt{6}}(2 r r g-r g r-g r r) \\
& \chi_{b 2}^{c 1}=\frac{1}{\sqrt{6}}(2 r r b-r b r-b r r) \\
& \chi_{b 3}^{c 1}=\frac{1}{\sqrt{6}}(r g g+g r g-2 g g r) \\
& \chi_{b 4}^{c 1}=\frac{1}{\sqrt{12}}(2 r g b-r b g+2 g r b-g b r-b r g-b g r) \\
& \chi_{b 5}^{c 1}=\frac{1}{\sqrt{4}}(r b g-g b r+b r g-b g r) \\
& \chi_{b 6}^{c 1}=\frac{1}{\sqrt{6}}(r b b+b r b-2 b b r) \\
& \chi_{b 7}^{c 1}=\frac{1}{\sqrt{6}}(2 g g b-g b g-b g g) \\
& \chi_{b 8}^{c 1}=\frac{1}{\sqrt{6}}(g b b+b g b-2 b b g)
\end{aligned}
$$

$\chi_{b 1}^{c 2}=\frac{1}{\sqrt{2}}(r g r-g r r)$

$\chi_{b 2}^{c 2}=\frac{1}{\sqrt{2}}(r b r-b r r)$

$\chi_{b 3}^{c 2}=\frac{1}{\sqrt{2}}(r g g-g r g)$

$\chi_{b 4}^{c 2}=\frac{1}{\sqrt{4}}(r b g+g b r-b r g-b g r)$

$\chi_{b 5}^{c 2}=\frac{1}{\sqrt{12}}(2 r g b+r b g-2 g r b-g b r-b r g+b g r)$

$\chi_{b 6}^{c 2}=\frac{1}{\sqrt{2}}(r b b-b r b)$

$\chi_{b 7}^{c 2}=\frac{1}{\sqrt{2}}(g b b-b g b)$

$\chi_{b 8}^{c 2}=\frac{1}{\sqrt{2}}(g b b-b g b)$, 
where superscripts $c 1$ and $c 2$ represent that the color symmetries of quarks 1 and 2 are symmetric and antisymmetric, respectively. The color wave function of the color-octet $q \bar{q}$ cluster can be written as

$\chi_{m 1}^{c}=r \bar{b}, \quad \chi_{m 2}^{c}=-r \bar{g}, \quad \chi_{m 3}^{c}=g \bar{b}$

$\chi_{m 4}^{c}=\frac{1}{\sqrt{2}}(r \bar{r}-g \bar{g}), \quad \chi_{m 5}^{c}=\frac{1}{\sqrt{6}}(2 b \bar{b}-r \bar{r}-g \bar{g})$

$\chi_{m 6}^{c}=-b \bar{g}, \quad \chi_{m 7}^{c}=g \bar{r}, \quad \chi_{m 8}^{c}=b \bar{r}$

Then, the total color wave functions for the hidden-color channel is obtained by coupling the wave functions of two color-octet clusters according to the Clebsch-Gordan coefficients.

$$
\begin{aligned}
\chi^{c 1}= & \frac{1}{\sqrt{8}}\left(\chi_{b 1}^{c 1} \chi_{m 8}^{c}-\chi_{b 3}^{c 1} \chi_{m 6}^{c}-\chi_{b 2}^{c 1} \chi_{m 7}^{c}+\chi_{b 4}^{c 1} \chi_{m 4}^{c}\right. \\
& \left.-\chi_{b 7}^{c 1} \chi_{m 2}^{c}+\chi_{b 5}^{c 1} \chi_{m 5}^{c}-\chi_{b 6}^{c 1} \chi_{m 3}^{c}+\chi_{b 8}^{c 1} \chi_{m 1}^{c}\right) \\
\chi^{c 2}= & \frac{1}{\sqrt{8}}\left(\chi_{b 1}^{c 2} \chi_{m 8}^{c}-\chi_{b 3}^{c 2} \chi_{m 6}^{c}-\chi_{b 2}^{c 2} \chi_{m 7}^{c}+\chi_{b 4}^{c 2} \chi_{m 4}^{c}\right. \\
& \left.-\chi_{b 7}^{c 2} \chi_{m 2}^{c}+\chi_{b 5}^{c 2} \chi_{m 5}^{c}-\chi_{b 6}^{c 2} \chi_{m 3}^{c}+\chi_{b 8}^{c 2} \chi_{m 1}^{c}\right)
\end{aligned}
$$

Finally, we can acquire the total wave function by combining the wave functions of the orbital, spin, flavor and color parts together according to the quantum number of the pentaquark systems.

\section{RESULTS AND DISCUSSIONS}

In this work, we investigate the $S$-wave fully heavy pentaquark systems $c c c c \bar{c}$ and $b b b b \bar{b}$ in the framework of two quark models, the ChQM and the QDCSM. The quantum numbers of these systems are $J^{P}=1 / 2^{-}, 3 / 2^{-}$, and $5 / 2^{-}$.

To find out if there is any bound state in such fully heavy pentaquark systems, we do a dynamic bound-state calculation here. The single-channel calculation and the channel coupling are carried out. All the calculation results for $c c c c \bar{c}$ and $b b b b \bar{b}$ systems in two models are listed in Tables I and IV, respectively. The second column headed with channel denotes the physical contents of each channel; $E_{t h}$ and $E_{s c}$ represent the threshold and the energy of each channel. As for the result of the channel-coupling calculation, $E_{c c 1}$ stands for the coupling of only colorsinglet channels, and $E_{c c 2}$ stands for the coupling of both the color-singlet channels and the hidden-color channels. Besides, the subscript 8 is marked to represent the coloroctet channels, and the asterisk $*$ is added to the energy, which is lower than the threshold of the corresponding channel.

\section{A. Fully charm pentaqurks}

The energies of the fully charm pentaquark systems are listed in Table I, including both the single-channel and channel-coupling results. Obviously, the energies of every single channel are above the corresponding theoretical threshold in both of the models, which means that each color-singlet channel is unbound. The energy of each hidden-color channel is much higher than that of the color-singlet channel. After the channel-coupling calculation, the energy of the $J^{P}=1 / 2^{-}$state is lower than the threshold of $\Omega_{c c c} J / \psi$, the binding energy of which is $-0.2 \mathrm{MeV}$ in ChQM and -1.0 MeV in QDCSM. However, for the $J^{P}=3 / 2^{-}$state, the energy is still higher than the theoretical threshold of $\Omega_{c c c} \eta_{c}$, which indicates that there is no bound state lower than $\Omega_{c c c} \eta_{c}$ for the $J^{P}=3 / 2^{-}$system in both of the models. Besides, there is only one channel for the $J^{P}=5 / 2^{-}$state, and it is not bound, either.

Besides, from Table I, one may note that the mass difference between ChQM and QCDSM is around $110 \mathrm{MeV}$. This is mainly due to the different parameters used in two models. As we mentioned in Sec. II, the parameters we used in this work are taken from our previous work of Ref. [36] directly to avoid adjusting the parameters. The work of Ref. [36] is about the $N \Omega_{c c c}$ and $N \Omega_{b b b}$ systems, in which the parameters were determined by fitting the masses of both the light baryons and the heavy baryons. Since the $\sigma$ exchange interaction is used in ChQM but it is not used in QDCSM, the parameters for these two models are different. When extending to the fully

\begin{tabular}{|c|c|c|c|c|c|c|c|c|c|}
\hline \multirow[b]{2}{*}{$J^{P}$} & \multirow[b]{2}{*}{ Channel } & \multicolumn{4}{|c|}{ ChQM } & \multicolumn{4}{|c|}{ QDCSM } \\
\hline & & $E_{t h}$ & $E_{s c}$ & $E_{c c 1}$ & $E_{c c 2}$ & $E_{t h}$ & $E_{s c}$ & $E_{c c 1}$ & $E_{c c 2}$ \\
\hline \multirow[t]{3}{*}{$\frac{1}{2}-$} & $\Omega_{c c c} J / \psi$ & 8427.7 & 8428.9 & & $8427.5^{*}$ & 8537.4 & 8538.6 & & $8536.4^{*}$ \\
\hline & $\Omega_{c c c 8} \eta_{c 8}$ & & 8658.4 & & & & 8786.8 & & \\
\hline & $\Omega_{c c c 8} J / \psi_{8}$ & & 8739.8 & & & & 8897.5 & & \\
\hline \multirow[t]{3}{*}{$\frac{3}{2}-$} & $\Omega_{c c c} \eta_{c}$ & 8424.9 & 8426.8 & 8425.6 & 8425.4 & 8534.8 & 8536.8 & 8535.5 & 8535.0 \\
\hline & $\Omega_{c c c} J / \psi$ & 8427.7 & 8429.5 & & & 8537.4 & 8539.2 & & \\
\hline & $\Omega_{c c c 8} J / \psi_{8}$ & & 8616.3 & & & & 8728.1 & & \\
\hline$\frac{5-}{2}$ & $\Omega_{c c c} J / \psi$ & 8427.7 & 8429.8 & & & 8537.4 & 8539.4 & & \\
\hline
\end{tabular}
heavy systems, different masses are obtained. In this work, the masses of the fully heavy baryon $\Omega_{c c c}$ and $\Omega_{b b b}$ are,

TABLE I. The energies of the $c c c c \bar{c}$ pentaquark systems (in $\mathrm{MeV}$ ). 
respectively, 5069.1 and 15111.6 $\mathrm{MeV}$ in $\mathrm{ChQM}$ and 5134.6 and $15169.4 \mathrm{MeV}$ in QDCSM. When extending to the mesons, none of the parameters was readjusted. So, the masses of the mesons $\eta_{c}, J / \psi, \eta_{b}$, and $\Upsilon$ are not well reproduced here. They are, respectively, 3355.8, 3358.6, 10067.1, and 10067.3 MeV in ChQM and 3400.2, 3402.8, 10105.7, and 10105.9 MeV in QDCSM. To show the difference between ChQM and QCDSM in detail, we also study the contribution of each interaction term to the state. To save space, we take the $\Omega_{c c c} J / \psi$ system with $J^{P}=$ $1 / 2^{-}$as an example. The contributions of each interaction term to the $\Omega_{c c c}$ and $J / \psi$ in both of the models are shown in Table II, and those to the $\Omega_{c c c} J / \psi$ system with $J^{P}=1 / 2^{-}$ are shown in Table III, where the $V_{m}, V_{k}, V_{\text {con }}$, and $V_{\text {oge }}$ represent the mass, kinetic energy, confinement, and onegluon-exchange interactions, respectively.

From Table II, we can see that the value of the interaction of each term is different between two models, leading to the total mass of $\Omega_{c c c}$ in QDCSM being about $65 \mathrm{MeV}$ higher than that in ChQM and the total mass of $J / \psi$ in QDCSM being about $45 \mathrm{MeV}$ higher than that in ChQM. So, the threshold of the $\Omega_{c c c} J / \psi$ state in QDCSM is about $110 \mathrm{MeV}$ higher than that in ChQM. For the single-channel $\Omega_{c c c} J / \psi$, since it is unbound, the energy will approach to the threshold. Table III shows the contributions of each interaction term by the channel-coupling calculation. We can see that the energy in QDCSM is still about $110 \mathrm{MeV}$ higher than that in ChQM, but the binding energy is very similar in two models. In ChQM, the energy of the kinetic energy is $13.1 \mathrm{MeV}$ higher than the threshold, and the confinement is $13.3 \mathrm{MeV}$ lower than the threshold, which

TABLE II. The contributions of each interaction term to $\Omega_{c c c}$ and $J / \psi($ in $\mathrm{MeV}$ ).

\begin{tabular}{lrrrrr}
\hline \hline & \multicolumn{2}{c}{$\Omega_{c c c}$} & & \multicolumn{2}{c}{$J / \psi$} \\
\cline { 2 - 3 } \cline { 5 - 6 } & ChQM & QDCSM & & ChQM & QDCSM \\
\hline$V_{m}$ & 5232.0 & 5364.0 & & 3488.0 & 3576.0 \\
$V_{k}$ & 124.8 & 121.7 & 62.4 & 60.9 \\
$V_{\text {con }}$ & -159.2 & -224.4 & & -106.1 & -149.6 \\
$V_{\text {oge }}$ & -128.5 & -126.7 & & -85.7 & -84.5 \\
$V_{\text {total }}$ & 5069.1 & 5134.6 & & 3358.6 & 3402.8 \\
\hline \hline
\end{tabular}

TABLE III. The contributions of each interaction term to the $\Omega_{c c c} J / \psi$ system with $J^{P}=1 / 2^{-}$(in $\mathrm{MeV}$ ).

\begin{tabular}{lrrrrrrr}
\hline \hline & \multicolumn{3}{c}{ ChQM } & & \multicolumn{3}{c}{ QDCSM } \\
\cline { 2 - 3 } \cline { 6 - 7 } & \multicolumn{1}{c}{$E_{t h}$} & \multicolumn{1}{c}{$E_{c c 2}$} & \multicolumn{1}{c}{$B$} & & \multicolumn{1}{c}{$E_{t h}$} & \multicolumn{1}{c}{$E_{c c 2}$} & \multicolumn{1}{c}{$B$} \\
\hline$V_{m}$ & 8720.0 & 8720.0 & 0.0 & 8940.0 & 8940.0 & 0.0 \\
$V_{k}$ & 187.2 & 200.3 & 13.1 & & 182.6 & 195.1 & 12.5 \\
$V_{\text {con }}$ & -265.3 & -278.6 & -13.3 & -374.0 & -387.5 & -13.5 \\
$V_{\text {oge }}$ & -214.3 & -214.3 & 0.0 & -211.3 & -211.3 & 0.0 \\
$V_{\text {total }}$ & 8427.7 & 8427.5 & -0.2 & 8537.4 & 8536.4 & -1.0 \\
\hline \hline
\end{tabular}

leads to the total $0.2 \mathrm{MeV}$ binding energy for the $\Omega_{c c c} J / \psi$ system with $J^{P}=1 / 2^{-}$. The result is similar in the QDCSM. From this analysis, we can see that, although the values of energy are different in two models because of the different parameters used, the binding energies of two models are much similar.

To minimize the theoretical errors and to compare calculated results to the experimental data in the future, we shift the mass of the pentaquark system here. Generally, the mass of a molecular pentaquark can be written as $M^{\text {the }}=M_{1}^{\text {the }}+M_{2}^{\text {the }}+B$, where $M_{1}^{\text {the }}$ and $M_{2}^{\text {the }}$ stand for the theoretical masses of a baryon and a meson, respectively, and $B$ is the binding energy of this molecular state. To minimize the theoretical errors, we can shift the mass of molecular pentaquark to $M=M_{1}^{\exp }+M_{2}^{\exp }+B$, where the experimental values of a baryon and a meson are used. Since there is no experimental value of the fully heavy $\Omega_{c c c}$ and $\Omega_{b b b}$, we use the values predicted by the lattice QCD calculation [44], which are 4796 and $14366 \mathrm{MeV}$, respectively. Taking the fully charm state $\Omega_{c c c} J / \psi$ with $J^{P}=1 / 2^{-}$in ChQM as an example, the calculated mass of the pentaquark is $8427.5 \mathrm{MeV}$; then, the binding energy $B$ is obtained by subtracting the theoretical masses of $\Omega_{c c c}$ and $\quad J / \psi, \quad 8427.5-5069.1-3358.6=-0.2(\mathrm{MeV})$. Using the lattice QCD mass of $\Omega_{c c c}$ and the experimental mass of $J / \psi$, the mass of this pentaquark is $M=$ $4796+3096.9+(-0.2)=7892.7(\mathrm{MeV})$. The same approach is used in the QDCSM, and the mass of this pentaquark is $7891.9 \mathrm{MeV}$ through the calculation. So, we finally obtain a fully charm pentaquark state with $J^{P}=1 / 2^{-}$, the mass of which is 7891.9-7892.7 MeV.

In addition, to confirm the existence of the fully charm pentaquark state with $J^{P}=1 / 2^{-}$, the spatial distribution feature of this state is investigated. The relative wave function between $\Omega_{c c c}$ and $J / \psi$ as a function of their distance $r$ in both of the models is plotted in Fig. 1. To compare the result of the single channel and that of the channel coupling, both of them are shown in Fig. 1. It is

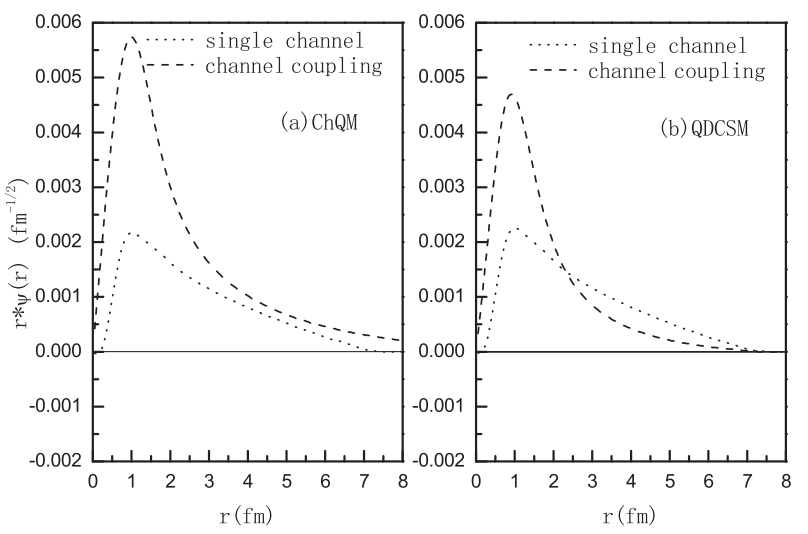

FIG. 1. The relative wave function of the fully charm pentaquark system with $J^{P}=1 / 2^{-}$. 
clear that the behavior of the relative wave function of the single channel stands for the unbound nature of the single $\Omega_{c c c} J / \psi$, while the one of the channel-coupling calculation indicates the bound property of this state. Therefore, the effect of the channel coupling is crucial for forming a bound state for the fully charm pentaquark system with $J^{P}=1 / 2^{-}$.

To understand the interaction between two heavy hadrons, we continue to study the effective potentials, which are shown in Figs. 2-4. The effective potential between two colorless clusters is defined as $V(S)=$ $E(S)-E(\infty)$, where $E(S)$ is the diagonal matrix element of the Hamiltonian of the system in the generating coordinate. From Figs. 2-4, we find that the results in both ChQM and QDCSM are similar. For the $J^{P}=1 / 2^{-}$ system (Fig. 2), one sees that the potential for the colorsinglet channel $\Omega_{c c c} J / \psi$ is attractive. However, the attraction is not strong enough to form a bound state. So, this single channel is unbound in the bound-state calculation. It

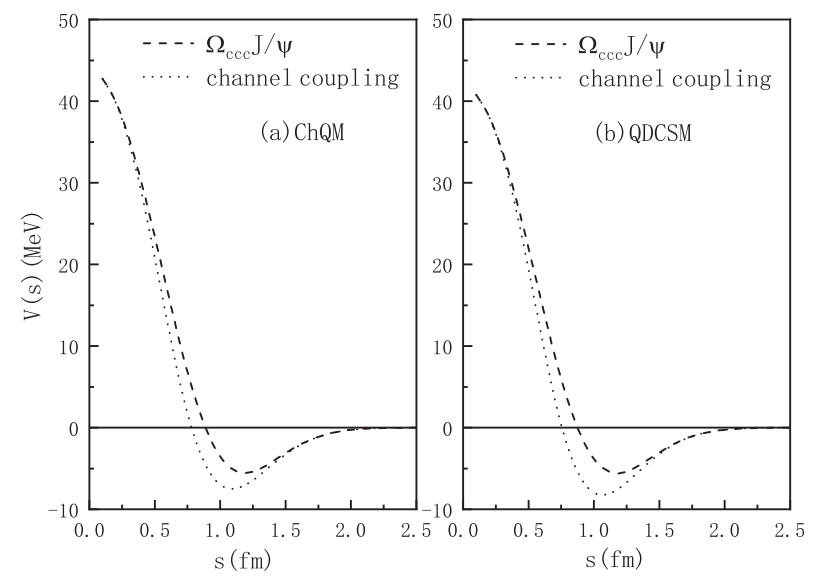

FIG. 2. The effective potentials of the fully charm system with $J^{P}=1 / 2^{-}$in ChQM and QDCSM.

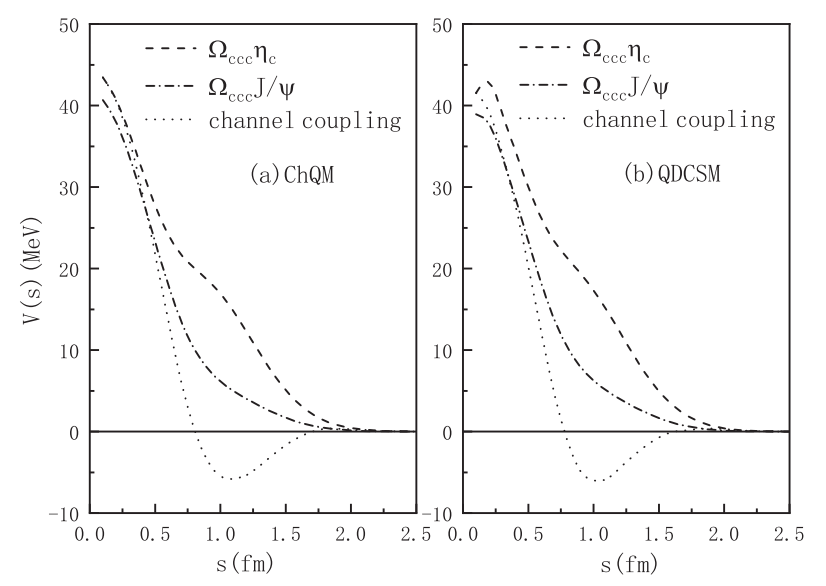

FIG. 3. The effective potentials of the fully charm system with $J^{P}=3 / 2^{-}$in ChQM and QDCSM.

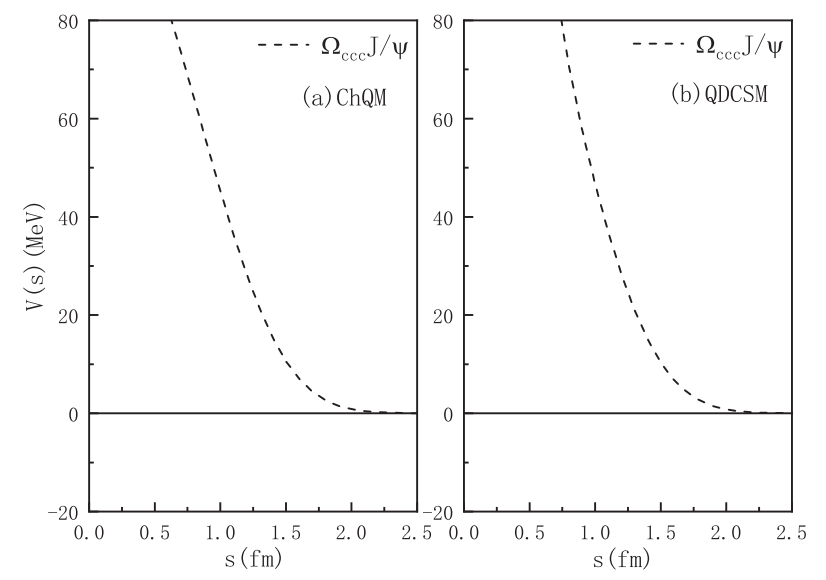

FIG. 4. The effective potentials of the fully charm system with $J^{P}=5 / 2^{-}$in ChQM and QDCSM.

is obvious that the attraction becomes larger by the channelcoupling calculation, which leads to the bound state. For the $J^{P}=3 / 2^{-}$system (Fig. 3 ), we can see that the effective potentials for the $\Omega_{c c c} \eta_{c}$ and $\Omega_{c c c} J / \psi$ are repulsive, so no bound states can be formed in these two channels. Although the potential is attractive by the channel coupling, it is not strong enough to form a bound state. So, we do not obtain the bound state for the $J^{P}=3 / 2^{-}$system. For the $J^{P}=5 / 2^{-}$system (Fig. 4), the potential for the $\Omega_{c c c} J / \psi$ is clearly repulsive. Besides, the repulsion increases greatly when the two hadrons $\Omega_{c c c}$ and $J / \psi$ get very close. This is due to the Pauli exclusion principle. Considering that the four $c$ quarks in the pentaquark system of $J^{P}=5 / 2^{-}$are identical particles, they have the same state in the orbital, flavor, and spin wave functions. But there are only three color states, which are red, green, and blue. Because of the Pauli exclusion principle, the system with $J^{P}=5 / 2^{-}$ containing these four identical quarks and an antiquark is not allowed to exist. That is why we cannot obtain the $\operatorname{ccc} \bar{c}$ pentaquark with $J^{P}=5 / 2^{-}$.

Besides, we also find the results in both of the models are almost the same; this is because the quarks are too heavy to run, resulting in the value of the quark delocalization parameter $\epsilon$ in QDCSM being close to 0. The color screening parameter in QDCSM is also very small because of the heavy quarks, which makes the difference of the confinement between two models be very small. So, both the effect of the quark delocalization and the color screening in QDCSM are very small in such a fully heavy system. Meanwhile, the $\sigma$ meson exchange is also inoperative in ChQM, which makes the coincident results of two models.

\section{B. Fully bottom pentaqurks}

In the previous discussion, the fully charm pentaquarks were investigated. We also extend the study to the fully bottom pentaquarks because of the heavy flavor symmetry. The numerical results are listed in Table IV. We find that 
TABLE IV. The energies of the $b b b b \bar{b}$ pentaquark systems (in MeV).

\begin{tabular}{|c|c|c|c|c|c|c|c|c|c|}
\hline \multirow[b]{2}{*}{$J^{P}$} & \multirow[b]{2}{*}{ Channel } & \multicolumn{4}{|c|}{ ChQM } & \multicolumn{4}{|c|}{ QDCSM } \\
\hline & & $E_{t h}$ & $E_{s c}$ & $E_{c c 1}$ & $E_{c c 2}$ & $E_{t h}$ & $E_{s c}$ & $E_{c c 1}$ & $E_{c c 2}$ \\
\hline \multirow[t]{3}{*}{$\frac{1-}{2}$} & $\Omega_{b b b} \Upsilon$ & 25178.9 & 25179.4 & & $25166.4^{*}$ & 25275.2 & 25275.6 & & $25259.0^{*}$ \\
\hline & $\Omega_{b b b 8} \eta_{b 8}$ & & 25308.7 & & & & 25388.6 & & \\
\hline & $\Omega_{b b b 8} \Upsilon_{8}$ & & 25380.1 & & & & 25496.0 & & \\
\hline \multirow[t]{3}{*}{$\frac{3}{2}-$} & $\Omega_{b b b} \eta_{b}$ & 25178.7 & 25179.4 & 25179.2 & $25166.3^{*}$ & 25275.0 & 25275.7 & 25275.5 & $25258.9^{*}$ \\
\hline & $\Omega_{b b b} \Upsilon$ & 25178.9 & 25179.6 & & & 25275.2 & 25275.5 & & \\
\hline & $\Omega_{b b b 8} \Upsilon_{8}$ & & 25272.8 & & & & 25335.3 & & \\
\hline$\frac{5-}{2}$ & $\Omega_{b b b} \Upsilon$ & 25178.9 & 25179.2 & & & 25275.2 & 25275.9 & & \\
\hline
\end{tabular}

the results are similar to the fully charm pentaquarks. All the color-singlet channel is unbound, and the energy of the hidden-color channel is much higher than the color-singlet channel. After the channel-coupling calculation, a bound state is obtained for the $J^{P}=1 / 2^{-}$system, with the binding energy of $-12.5 \mathrm{MeV}$ in ChQM and $-16.2 \mathrm{MeV}$ in QDCSM. By performing the mass shift, we finally obtain the mass of this pentaquark, which is 23810.1-23813.8 MeV. Besides, the state with $J^{P}=3 / 2^{-}$ is also bound with the help of channel coupling. The binding energy is $-12.4 \mathrm{MeV}$ in $\mathrm{ChQM}$ and $-16.1 \mathrm{MeV}$ in QDCSM. By shifting the mass, the mass of the fully bottom pentaquark state with $J^{P}=3 / 2^{-}$is $23748.2-23752.3 \mathrm{MeV}$.

Comparing with the results of the fully charm pentaquarks, we find that the binding energy of the fully bottom system is higher than that of the fully charm system. This is mainly due to the kinetic energy and the confinement interaction between the baryon and the meson. We also calculate the contribution of each interaction term to the fully bottom system. To compare with the fully charm system, we show the results of the $J^{P}=1 / 2^{-} \Omega_{b b b} \Upsilon$ system with channel-coupling calculation in Table V. From Tables III and V, we can see that the contribution of the positive kinetic energy for the $\Omega_{b b b} \Upsilon$ system is smaller than that for the $\Omega_{c c c} J / \psi$ system. This is because the bottom quark is much heavier than the charm quark. Besides, because the bottom quark is too heavy to run, the distance between bottom quarks will smaller than the one between charm quarks, which leads to the contribution of the negative confinement interaction for the $\Omega_{b b b} \Upsilon$ system

TABLE V. The contributions of each interaction term to the $\Omega_{b b b} \Upsilon$ system with $J^{P}=1 / 2^{-}$(in $\mathrm{MeV}$ ).

\begin{tabular}{lrrrrrrr}
\hline \hline & \multicolumn{3}{c}{ ChQM } & & \multicolumn{3}{c}{ QDCSM } \\
\cline { 2 - 4 } \cline { 6 - 8 } & \multicolumn{1}{c}{$E_{t h}$} & \multicolumn{1}{c}{$E_{c c 2}$} & \multicolumn{1}{c}{$B$} & & $E_{t h}$ & \multicolumn{1}{c}{$E_{c c 2}$} & \multicolumn{1}{c}{$B$} \\
\hline$V_{m}$ & 25500.0 & 25500.0 & 0.0 & & 25705.0 & 25705.0 & 0.0 \\
$V_{k}$ & 64.0 & 71.7 & 7.7 & & 63.5 & 72.1 & 8.6 \\
$V_{\text {con }}$ & -265.3 & -285.4 & -20.1 & & -374.0 & -398.6 & -24.6 \\
$V_{\text {oge }}$ & -119.8 & -119.9 & -0.1 & -119.3 & -119.5 & -0.2 \\
$V_{\text {total }}$ & 25178.9 & 25166.4 & -12.5 & & 25275.2 & 25259.0 & -16.2 \\
\hline \hline
\end{tabular}

being larger than that for the $\Omega_{c c c} J / \psi$ system. That is why the binding energy of the fully bottom pentaquark is higher than that of the fully charm pentaquark.

Additionally, the effective potentials of the fully bottom pentaquark systems in both ChQM and QDCSM are also

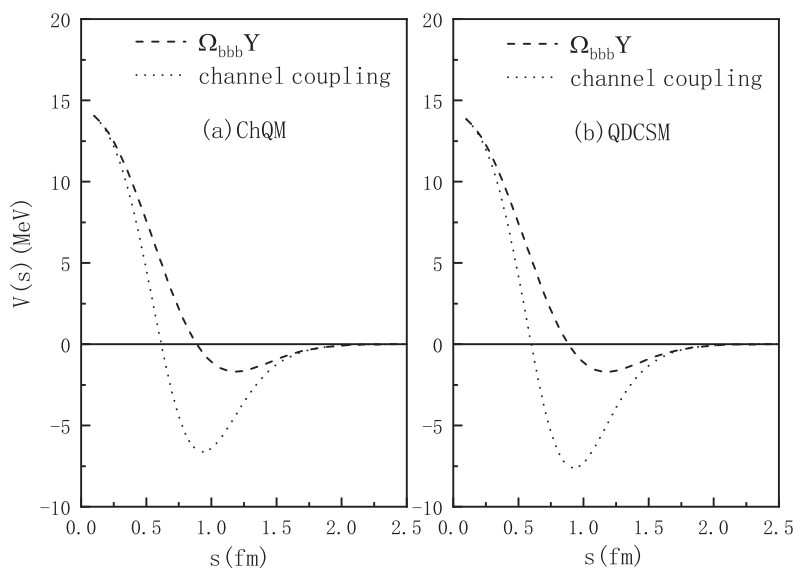

FIG. 5. The effective potentials of the fully bottom system with $J^{P}=1 / 2^{-}$in ChQM and QDCSM.

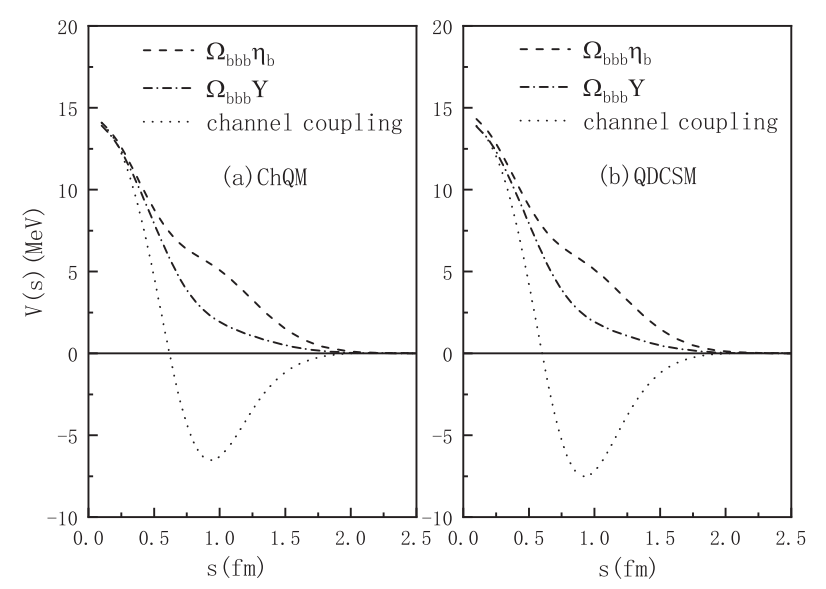

FIG. 6. The effective potentials of the fully bottom system with $J^{P}=3 / 2^{-}$in ChQM and QDCSM. 


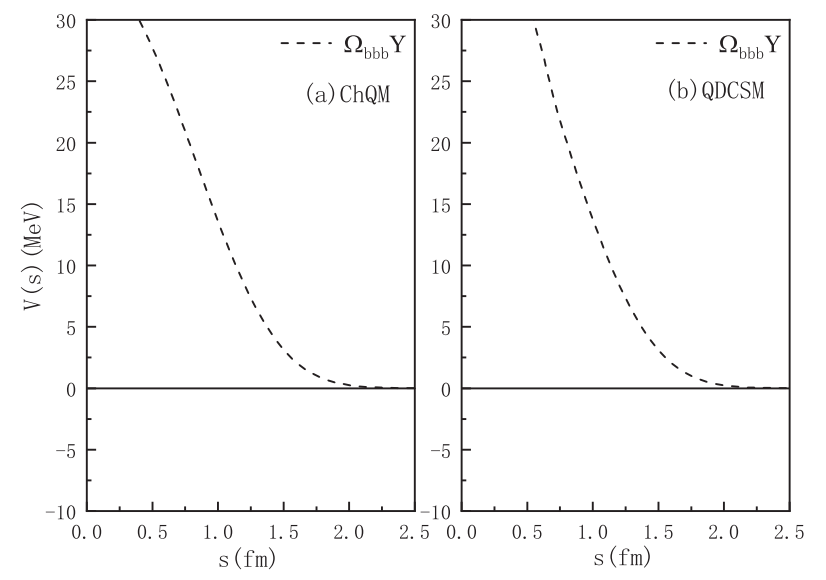

FIG. 7. The effective potentials of the fully bottom system with $J^{P}=5 / 2^{-}$in ChQM and QDCSM.

studied and are shown in Figs. 5-7. Obviously, the results are also similar to the fully charm pentaquark systems.

\section{CONCLUSIONS}

In this work, we systematically investigate the low-lying fully heavy pentaquark systems $c c c c \bar{c}$ and $b b b b \bar{b}$ in two quark models: ChQM and QDCSM. The dynamic boundstate calculation is carried out to search for any bound state in the fully heavy systems. Both the single-channel and the channel-coupling calculations are performed to explore the effect of the multichannel coupling. Meanwhile, the relative wave function of the fully charm pentaquark state is studied to confirm the existence of the bound state. Besides, an adiabatic calculation of the effective potentials is added to explore the interaction between the heavy baryon and the heavy meson.

The numerical results show that the effect of the channel coupling is important for forming a bound state of the fully heavy pentaquark system and the conclusions are consistent in both ChQM and QDCSM. With the help of the channel coupling, we obtain three fully heavy pentaquarks, which are the fully charm pentaquark with $J^{P}=1 / 2^{-}$and the mass of 7891.9-7892.7 MeV, the fully bottom pentaquark with $J^{P}=1 / 2^{-}$and the mass of 23810.1-23813.8 MeV, and the fully bottom pentaquark with $J^{P}=3 / 2^{-}$and the mass of $23748.2-23752.3 \mathrm{MeV}$. All these fully heavy pentaquark states are worth looking for in future experiments.

In Ref. [25], the study of the fully heavy pentaquarks in the CMI model found two $c c c c \bar{c}$ states with $J^{P}=1 / 2^{-}$ and $3 / 2^{-}$, respectively, but the masses of them were above the thresholds of the $\Omega_{c c c} J / \psi$ and $\Omega_{c c c} \eta_{c}$, respectively. The channel coupling may bring down the masses of these states. In the framework of QCD sum rules, both the $c c c c \bar{c}$ state and $b b b b \bar{b}$ state are predicted in two works, Refs. [26,27]. However, the masses and the structure of these states are different in two works. More theoretical studies and experimental efforts, which may shed more light on the nature of the fully heavy pentaquark states, are expected.

\section{ACKNOWLEDGMENTS}

This work is supported partly by the National Natural Science Foundation of China under Contracts No. 11675080, No. 11775118, and No. 11535005.
[1] R. Aaij et al. (LHCb Collaboration), Sci. Bull. 65, 1983 (2020).

[2] Z. G. Wang, Chin. Phys. C 44, 113106 (2020).

[3] M. S. Liu, F. X. Liu, X. H. Zhong, and Q. Zhao, arXiv: 2006.11952.

[4] P. Lundhammar and T. Ohlsson, Phys. Rev. D 102, 054018 (2020).

[5] Q. F. Lü, D. Y. Chen, and Y. B. Dong, Eur. Phys. J. C 80, 871 (2020).

[6] M. Karliner and J. L. Rosner, Phys. Rev. D 102, 114039 (2020).

[7] G. Yang, J. Ping, L. He, and Q.Wang, arXiv:2006.13756.

[8] X. Jin, Y. Xue, H. Huang, and J. Ping, Eur. Phys. J. C 80, 1083 (2020).

[9] R. M. Albuquerque, S. Narison, A. Rabemananjara, D. Rabetiarivony, and G. Randriamanatrika, Phys. Rev. D 102, 094001 (2020).

[10] J. Sonnenschein and D. Weissman, Eur. Phys. J. C 81, 25 (2021).
[11] J. M. Richard, Sci. Bull. 65, 1954 (2020).

[12] K. T. Chao and S. L. Zhu, Sci. Bull. 65, 1952 (2020).

[13] T. Goldman, K. Maltman, G. J. Stephenson, K. E. Schmidt, and F. Wang, Phys. Rev. Lett. 59, 627 (1987).

[14] Z. Y. Zhang, Y. W. Yu, C. R. Ching, T. H. Ho, and Z. D. Lu, Phys. Rev. C 61, 065204 (2000).

[15] T. Iritani et al., Phys. Lett. B 792, 284 (2019).

[16] S. Gongyo et al., Phys. Rev. Lett. 120, 212001 (2018).

[17] H. Huang, J. Ping, X. Zhu, and F. Wang, arXiv:2011.00513.

[18] P. Junnarkar and N. Mathur, Phys. Rev. Lett. 123, 162003 (2019).

[19] Y. Lyu, H. Tong, T. Sugiura, S. Aoki, T. Doi, T. Hatsuda, J. Meng, and T. Miyamoto, Phys. Rev. Lett. 127, 072003 (2021).

[20] R. Aaij et al. (LHCb Collaboration), Phys. Rev. Lett. 115, 072001 (2015).

[21] R. Aaij et al. (LHCb Collaboration), Phys. Rev. Lett. 122, 222001 (2019).

[22] H. X. Chen, W. Chen, X. Liu, and S. L. Zhu, Phys. Rep. 639, 1 (2016). 
[23] Y. R. Liu, H. X. Chen, W. Chen, X. Liu, and S. L. Zhu, Prog. Part. Nucl. Phys. 107, 237 (2019).

[24] G. Yang, J. L. Ping, and J. Segovia, Symmetry 12, 1869 (2020).

[25] H. T. An, K. Chen, Z. W. Liu, and X. Liu, Phys. Rev. D 103, 074006 (2021).

[26] J. R. Zhang, Phys. Rev. D 103, 074016 (2021).

[27] Z. G. Wang, Nucl. Phys. B 973, 115579 (2021).

[28] N. Ishii, S. Aoki, and T. Hatsuda, Phys. Rev. Lett. 99, 022001 (2007).

[29] C. Alexandrou, P. De Forcrand, and A. Tsapalis, Phys. Rev. D 65, 054503 (2002).

[30] F. Okiharu, H. Suganuma, and T. T. Takahashi, Phys. Rev. Lett. 94, 192001 (2005).

[31] A. Valcarce, H. Garcilazo, F. Fernández, and P. Gonzalez, Rep. Prog. Phys. 68, 965 (2005) and references therein.

[32] F. Wang, G. H. Wu, L. J. Teng, and J. T. Goldman, Phys. Rev. Lett. 69, 2901 (1992).

[33] L. Z. Chen, H. R. Pang, H. X. Huang, J. L. Ping, and F. Wang, Phys. Rev. C 76, 014001 (2007).
[34] M. Chen, H. X. Huang, J. L. Ping, and F. Wang, Phys. Rev. C 83, 015202 (2011).

[35] J. Vijande, F. Fernandez, and A. Valcarce, J. Phys. G 31, 481 (2005).

[36] H. Huang, J. Ping, and F. Wang, Phys. Rev. C 101, 015204 (2020).

[37] H. X. Huang, C. R. Deng, J. L. Ping, and F. Wang, Eur. Phys. J. C 76, 624 (2016).

[38] H. X. Huang and J. L. Ping, Phys. Rev. D 99, 014010 (2019).

[39] J. A. Wheeler, Phys. Rev. 52, 1083 (1937).

[40] M. Kamimura, Prog. Theor. Phys. Suppl. 62, 236 (1977).

[41] D. L. Hill and J. A. Wheeler, Phys. Rev. 89, 1102 (1953).

[42] J. J. Griffin and J. A. Wheeler, Phys. Rev. 108, 311 (1957).

[43] M. M. Xu, M. Yu, and L. S. Liu, Phys. Rev. Lett. 100, 092301 (2008).

[44] Z. S. Brown, W. Detmold, S. Meinel, and K. Orginos, Phys. Rev. D 90, 094507 (2014). 